\title{
Por un modelo de patrimonialización del paisaje agroecológico urbano en América Latina
}

\begin{abstract}
Paloma Gallegos Tejeda ${ }^{1}$
Pedro Lina Manjarrez ${ }^{2}$

Resumen: El objetivo del presente trabajo es visualizar la patrimonialización de lo agroecológico urbano para su reconocimiento como un bien material e inmaterial perteneciente a los recursos paisajísticos de la ciudad. Esto reviste una importante lucha social, ambiental, económica y cultural por la autosuficiencia alimentaria ante las desigualdades del desarrollo humano en las urbes de América Latina. Se considera importante la búsqueda de valores del Paisaje Agroecológico para la afirmación y permanencia del sistema agroalimentario en la ciudad, a través de huertos y proyectos productivos comunitarios con diferentes actores sociales involucrados. Se exploran por medio de la revisión documental y la observación directa en México, Colombia y Brasil. Las experiencias, una de cada país, señalan la diversidad de elementos, generales y contextuales, que estructuran sus valores paisajísticos. Resulta que existen características fundamentales para la acción de patrimonialización que bien podrían sustentarse en el marco legal e inserción de los planes y programas de desarrollo urbano sostenible, y abre perspectivas para la realización de planes de manejo de unidades paisajísticas agroecológicas urbanas que generen infraestructura y dinámicas sociales que tienden a mejorar cuestiones alimentarias.
\end{abstract}

Palabras clave: paisaje agroecológico; patrimonio; agroalimentación; conservación.

\section{Para um modelo de patrimonialização de uma paisagem agroecológica urbana na América Latina}

Resumo: O objetivo do presente trabalho é visualizar a patrimonialização da agroecologia urbana para seu reconhecimento como um bem material e imaterial, pertencente aos Recursos da Paisagem. Isso é importante para a luta social, ambiental, econômica e cultural pela auto-suficiência alimentar diante das desigualdades no desenvolvimento humano nas cidades latino-americanas. Considera-se importante a busca dos valores da Paisagem Agroecológica pelo reconhecimento e permanência do sistema agro-alimentar na cidade, isto pelos diferentes atores sociais envolvidos. São exploradas por meio de revisão documental e observação direta no México, Colômbia e Brasil. As experiências de agricultura urbana, uma de cada país, mostram a diversidade de elementos, generais e contextuais que estruturam seus valores da paisagem. Resulta, portanto, que existem características de um caminho ou padrão para a ação de patrimonialização que poderiam basear-se no marco legal e na inserção nos Planos e Programas de Desenvolvimento Urbano Sustentável, e abrem perspectivas para a realização dos Planos de Manejo das Unidades do Paisagem Agroecológico que geram infraestrutura e dinâmica social que tendem a melhorar as questões alimentares.

Palavras-chave: paisagem agroecológica; patrimônio; agroalimentação; conservação.

\section{For a patrimonialization model of the urban agroecological landscape in Latin America}

\begin{abstract}
The aim of this work is to visualize the patrimonialization of urban agroecological heritage to recognize tangible and intangible Landscape Resources appreciation thereof. This is important for social, environmental, economic and cultural struggle for food self-sufficiency in the face of inequalities in human development in Latin American cities. The recognition of Agroecological Landscape's values considers the presence of the agri-food system, community orchards in the city by the different social actors involved. The review is from documents and directly observation of urban agriculture experiences in Mexico, Colombia and Brazil that explore evidence of the landscape. Each experience has the diversity of general and contextual elements that gives structure to values landscape. As a result, there are fundamental features of pattern for the patrimonialization action. The legal framework and insertion in the Sustainable Urban Development Plans and Programs, could be based on opens perspectives for the development of Management Plans of Agroecological Landscape Units. The agroecological landscape generate social infrastructure and social dynamics interaction that tend to improve food issues.
\end{abstract}

Keywords: agroecological landscape; heritage; agri-food systems; conservation.

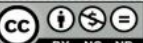

DOI: https://doi.org/10.26512/patryter.v4i8.31299

Como citar este artigo: Gallegos Tejeda, P., \& Lina Manjarrez, P. (2021). Por un modelo de patrimonialización del paisaje agroecológico urbano en América Latina. PatryTer - Revista Latinoamericana e Caribenha de Geografia e Humanidades, 4(8), 156171. DOI: https://doi.org/10.26512/patryter.v4i8.31299

Recebido: 02 de maio de 2020. Aceite: 03 de outubro de 2020. Publicado: 01 de setembro de 2021.

${ }^{1}$ Candidata a doctora por el Instituto Politécnico Nacional (IPN, México). ORCID: https://orcid.org/0000-0003-2126-

6716. E-mail: palomagallegos@yahoo.com.mx.

2 Profesor Investigador del Instituto Politécnico Nacional (IPN, México). ORCID: https:/ / orcid.org/0000-0001-6532-9374.

E-mail: linapedro@hotmail.com. 


\section{Introducción}

El paisaje patrimonial agrícola urbano se reconoce a partir de entender la necesidad de producir y acceder a alimentos frescos y naturales en la ciudad. Lo anterior se pretende traducir en la diversificación de la dieta familiar, la mejora de la economía doméstica, la gestión ambiental y el rescate de espacios públicos con perfil productivo que forjen un panorama donde se visualice la soberanía alimentaria urbana a partir de generar un patrimonio agroecológico urbano.

Mooney e ETC Group (2019, p. 47) establecen que "la soberanía alimentaria, incluyendo la agroecología campesina, debe ser la base para crear políticas alimentarias nacionales, con equidad de género y resiliencia comunitaria, fortaleciendo la cooperación en lugar de la competencia”. Ante la implementación de la Agricultura 4.0 por las transnacionales agroindustriales que fomenta el monocultivo, la monodieta y la tecnificación del campo, el autor opina que, "no se rechaza a la tecnología, aunque la prioridad son las comunidades, fomentar la agrobiodiversidad y la alimentación culturalmente apropiada" (Mooney \& ETC Group, 2019, p. 50).

La problemática de la habilitación del paisaje agroecológico urbano se establece a partir de observar la conformación del panorama urbano donde encontramos, en muchos casos, unidades habitacionales de alta densidad y falta de servicios donde prevalecen los intereses inmobiliarios en lugar del buen vivir y la alimentación humana, arriesgando los espacios públicos con viabilidad para producir comestibles. Los espacios públicos podrían resultar un recurso patrimonial del paisaje agroecológico urbano, cómo afirma Erlij (2004, p. 1) cuando menciona que "el espacio público es el motor de recuperación de un área deprimida y un elemento estructural para generar la identificación de un barrio. En consecuencia, para generar una rehabilitación de los barrios patrimoniales, actuar en los espacios públicos es fundamental".

Desde esta mirada se constata que existen intentos por desarrollar una agricultura urbana que pudiese ofrecer una perspectiva sostenible para la producción y acceso a alimentos con valores sociales, ambientales y económicos. Tales son los casos de la periferia metropolitana de Guadalajara, México; Florianópolis, Brasil; y Bogotá, Colombia. De estas experiencias se han obtenido aspectos que apuntan hacia una agricultura agroecológica amable con la tierra, la salud y la participación en la búsqueda del beneficio familiar y comunitario.
América Latina vive experiencias relacionadas con el intento de realizar una agricultura urbana que ofrezca alternativas de producción alimentaria con productos agrícolas ecológicos accesibles a la economía y salud de los habitantes de la ciudad.

La Organización de las Naciones Unidas para la Agricultura y la Alimentación - FAO (1996) define la agricultura urbana como:

\section{(...) la producción de alimentos dentro de los confines de las ciudades: en los patios, terrazas, buertos comunitarios y buertas de frutales, así como en espacios públicos o no aprovechados. Incluye operaciones comerciales que producen alimentos en invernaderos y en espacios al aire libre, pero en la mayoría de los casos se trata de una actividad en pequeña escala y dispersa por toda la ciudad.}

En el 2006, un estudio desarrollado por el Centro Internacional de Investigaciones para el Desarrollo en 10 países de Latinoamérica (Argentina, Brasil, Cuba, Ecuador, México, Honduras, Colombia, República Dominicana, Perú y Uruguay) explica que las municipalidades reconocen los beneficios potenciales de la agricultura urbana como una actividad en expansión y aunque no asegura la autosuficiencia alimentaria de las ciudades, "pueden volverlas más verdes, limpias, saludables y sostenibles" (FAO, 2006, p. 78). Añade Mougeot (2006, p. 38) que a pesar de todos sus atributos "la agricultura urbana en la planeación de la administración pública es prácticamente ignorada".

Por su parte, la FAO (2010) informa que los hogares urbanos involucrados en actividades de agricultura urbana tienen mayor acceso a alimentos nutritivos y frescos aumentando el consumo de frutas, hortalizas y en algunos casos, carne. Además, adquieren una dieta más diversificada y saludable. Y explica algunos elementos de sustentabilidad de la agricultura urbana enfatizando los procesos organizativos, la convivencia, el acercamiento con la naturaleza y la agrobiodiversidad. Además, caracteriza a la agricultora y al agricultor urbanos, como los protagonistas de la actividad, quienes desarrollan una serie de habilidades técnicas y socioeconómicas que benefician a sus propios hogares y a la comunidad.

En relación con la seguridad alimentaria, Toledo (2014, p. 14) plantea cómo México refleja lo que está ocurriendo en otros países de Latinoamérica; este pierde su autosuficiencia alimentaria por la incapacidad para autoabastecerse no por el "incremento de su población, ni de las 
Paloma Gallegos Tejeda

Pedro Lina Manjarrez
Por un modelo de patrimonialización

del paisaje agroecológico urbano en América Latina formas agrarias de organización y producción, sino de la expoliación de los productores, sus saberes y sus medios de producción (ecosistemas)". La cuestión es la aplicación reiterada de un modelo tecnológico que ignora las condiciones biológicas, ecológicas, ni culturales del país, lo que tiene efecto sobre la biodiversidad, los productores y la producción de alimentos. Además, el autor habla que "la pérdida de la autosuficiencia alimentaria en los ámbitos familiar, local y regional es efecto de un modelo típicamente centralizador, basado en la transferencia de capital de la periferia hacía el centro" (Toledo, 2014, p. 19).

El Grupo de Acción sobre Erosión, Tecnología y Concentración - ETC Groupii, a nivel nacional, en su informe de 2017, “¿Quién nos alimentará?”, aproxima la cadena alimentaria industrial a la red alimentaria campesina, aportando pruebas para demostrar que, mientras los campesinos - incluyendo agricultores, pescadores, cultivadores urbanos, trabajadores agrícolas, pastores y cosechadores forestales - producen la mayor parte de la comida que alimenta a más de dos tercios de la población del mundo, la cadena alimentaria industrial ocupa el $75 \%$ de la tierra cultivable y utiliza la mayor parte de los combustibles fósiles y productos químicos atribuidos a la agricultura (ETC Group, 2017).

$\mathrm{Al}$ explorar la presencia de la agricultura en la ciudad las expectativas de su alcance son muy diversas. La posibilidad de ser una alternativa productiva, y en muchos de los casos una opción para mejorar las condiciones de vida, se relaciona con la permanencia y consolidación de la actividad a partir de garantizar el espacio para siembra, los recursos como el agua, la tierra, las semillas, las herramientas, por mencionar algunos, y la mano campesina y otros interesados con capacidades asimismo compromiso para el cuidado de la tierra y la alimentación. En relación con eso se buscan las experiencias latinoamericanas que nos muestren el patrimonio generado por el paisaje de la agricultura urbana.

La metodología se desarrolla con el análisis y síntesis documental para alcanzar el objetivo de obtener información relevante en la revisión de publicaciones, planes y programas de temas de agricultura urbana, paisaje y agroecología como: (a) Convenio Europeo del Paisaje 2000 (Italia); (b) Plan Municipal de Desarrollo y Gobernanza del Municipio de Tlajomulco de Zúñiga 2018-2021 (Jalisco, México); (c) Plan de Desarrollo Bogotá sin Indiferencia 2004-2008 (Colombia); (d) Programa de gestão comunitária de resíduos orgânicos: o caso do Projeto Revolução dos Baldinhos 2013 (Brasil); (e) Informes de la Organización de las Naciones Unidas para la Agricultura y la Alimentación (FAO, 1996, 2006, 2010); entre otros.

La observación directa en campo así como las reuniones de trabajo, de capacitación, desarrollo del diseño y habilitación del huerto Lomas del Sur "Mercado Orgánico" en Tlajomulco de Zúñiga, Jalisco, México durante el 2017; el inicio del Proyecto Revolução dos Baldinhos, en el 2009; además, la visita al Jardín Botánico de Bogotá, en 2017.

La relación conceptual que se plantea entre el paisaje de la agricultura urbana y la agroecología muestra las pautas para entender las técnicas y principios que se utilizan para el desarrollo de esta práctica. Lo que nos lleva a reconocer los elementos del paisaje agroecológico urbano para establecer su valor patrimonial. En este sentido observar la infraestructura generada y los saberes agrícolas que se desarrollan e intercambian nos acercan al patrimonio natural y cultural agroalimentario. Lo anterior permite proponer un modelo de patrimonialización del paisaje agroecológico urbano.

Por lo tanto, el objetivo es identificar los elementos patrimoniales para la conservación del paisaje agroecológico urbano en tres casos de programas de desarrollo de agricultura urbana en Latinoamérica. Estas experiencias muestran la perspectiva de los recursos del paisaje agroecológico urbano para ser patrimonializados como antesala del reconocimiento en los ámbitos social, ambiental, económico y cultural de los elementos paisajísticos agroecológicos urbanos en su valor construido como la infraestructura y espacios donde se ven los bienes materiales que se generan y en el valor resiliente de los elementos inmateriales que en el sentido ecológico buscan la agrobiodiversidad, el control de plagas, además de la recuperación de suelos por citar algunos ejemplos, y en el sentido social, la organización, el desarrollo de capacidades y la búsqueda de procesos autogestivos.

Los tres estudios de caso, Huerto Lomas del Sur de Tlajomulco de Zúñiga, México, la Revolução dos Baldinhos de Florianópolis, Brasil, y las Camas Productivas de agricultura urbana en el Jardín Botánico de Bogotá, presentan constructos conceptuales de la relación paisaje-ciudadagricultura-ecología-patrimonio, con la idea de esclarecer el rol de la producción alimentaria de las comunidades agrarias urbanas y sus valores en el contexto de un paisaje transformado como un recurso paisajístico agroecológico (Gobierno de Bogotá, 2019). 
Paloma Gallegos Tejeda

Pedro Lina Manjarrez

\section{Desarrollo}

\subsection{Agricultura urbana como patrimonio paisajístico agroecológico}

La agricultura urbana como patrimonio paisajístico se basa en la producción agroecológica pues los recursos que se utilizan para su desarrollo son inocuos y naturales para no causar perjuicio a la salud. Se apoya en las agriculturas familiar y campesina, ya que por la restricción de los espacios citadinos y la participación social se observan sistemas alimentarios diversos y alternativos.

A partir de la integración de la agricultura urbana con la agroecología se visualiza una perspectiva sistémica de unidades urbanas vivas de producción alimentaria que restablecen espacios metropolitanos como proveedores de alimentos frescos. Al reconocerlo como paisaje agroecológico urbano la interacción socio ambiental se enriquece al generar un recurso paisajístico natural y cultural de la tierra que llega hasta la mesa de los involucrados.

Estas ideas se relacionan con lo que plantea Silva (2009, p. 121) cuando señala que los "paisajes agrarios son considerados como las configuraciones formales, particulares e irrepetibles en cada ámbito, de las combinaciones entre los sistemas de cultivo, las condiciones físicas y las estructuras sociales" y hace referencia con esto que el patrimonio agrícola no debería de verse solo como herencia sino como pertenencia y legado.

Silva (2009) agrega que el patrimonio agrícola se constituye a partir de la interacción entre el ser humano y la tierra, sustentado por lo agrícola o ganadero, interacción que da lugar al patrimonio inmaterial en constante cambio, y es a través de la historia de la agroalimentación que se consigue apreciar su valor social, ecológico y económico. Tal referencia, se refuerza con lo que plantea Castillo (2014, p. 109): "el patrimonio agrario es el conjunto de bienes naturales y culturales, materiales e inmateriales, generados o aprovechados por la actividad agraria a lo largo de la historia".

$\mathrm{Si}$ bien el patrimonio agroecológico, se relaciona con el patrimonio agrario y no con el patrimonio urbano; este se establece como un bien material social y cultural que conecta al paisaje, en un sentido, con la necesidad de su conservación. Al respecto Silva (2008, p. 2) plantea que:

(...) se entiende como patrimonio agrario todo aquel legado relacionado con la herencia histórica de la explotación agropecuaria, entendida ésta en un
Por un modelo de patrimonialización del paisaje agroecológico urbano en América Latina

sentido amplio (áreas de cultivo, dehesas, pastizales, plantaciones forestales), bien sea de carácter material (paisajes, edificios relacionados con la producción y la transformación de los productos de la agricultura, infraestructuras y equipamientos agrarios, determinados tipos de hábitat rural) o etnográfico (oficios, artesanias, folklore).

Silva $(2008,2009)$ vincula al patrimonio agrario con el hábitat rural y Castillo (2014) con un amplio ámbito. Ambos planteamientos permiten relacionarlo con el patrimonio urbano y el paisaje agroecológico urbano que lo articula con el campo pues, en esencia, al construirse genera una infraestructura necesaria para su funcionamiento.

$\mathrm{Al}$ respecto Hajba (2017, p. 303) señala que cultivar alimentos en la ciudad es un acto político, ya que además de “(...) producir alimentos saludables y moldear el entorno, construye comunidad. La experiencia es creer juntos, pensar juntos, aprender juntos a cooperar, porque el huerto y la ciudad son una utopía".

Altieri y Nihcolls (2000, p. 77) definen el enfoque agroecológico a través de los ecosistemas agrícolas como unidades fundamentales y "en estos sistemas, los ciclos minerales, las transformaciones de la energía, los procesos biológicos y las relaciones socioeconómicas son investigados y analizados como un todo". Entonces la acción agrícola se aborda desde un enfoque holístico que "va más allá de un punto de vista unidimensional de los agroecosistemas (su genética, edafología y otros) para abordar un entendimiento de los niveles ecológicos y sociales de coevolución, estructura y función" (Altieri \& Nihcolls, 2000, p. 80). La clave para funcionar es "la promoción de la biodiversidad en agroecosistemas para el rediseño por la biota asociada que garantiza una mejor polinización y regulación de plagas, enfermedades y malezas" (Altieri \& Nihcolls, 2000, p.81).

A este aspecto, lo que se plantea es que el paisaje de la agricultura urbana busca articularse con los sistemas de producción de alimentos y los recursos naturales que brindan los servicios ambientales desde la sustentabilidad social, ambiental, económico, cultural y ecológica. Esto se relaciona con lo planteado por la agricultura sustentable que para establecer "la seguridad alimentaria, erradicar la pobreza, conservar y proteger el ambiente y los recursos naturales, se debe alcanzar una agricultura ecológicamente íntegra, socialmente justa, culturalmente diversa y económicamente viable" (Altieri, 2010, p. 78). 
Paloma Gallegos Tejeda

Pedro Lina Manjarrez
Por un modelo de patrimonialización

del paisaje agroecológico urbano en América Latina
En este entendimiento, el sistema agroalimentario urbano se torna una parte compleja del propio paisaje urbano al tener limitado acercamiento a la tierra cultivable en la ciudad así como producir alimentos limpios y variados; además el acceso a los productos de la agricultura convencional parece ser la opción en la mayoría de los mercados junto con el cambio de uso de suelo sin planeación urbana sustentable. Esto, con las restricciones en la política pública que no puntualiza los bienes, ni la permanencia de las personas; tampoco la infraestructura necesaria para un proyecto integral de agricultura urbana acorde a la economía local y la conservación del medio ambiente, recursos necesarios en el territorio.

Rodríguez (2020, p. 98) plantea la valoración del territorio de excepción como patrimonio y forma de resistencia "a través de los sujetos, los productos de su trabajo y sus vínculos identitarios y sociales con el territorio", de esta forma ellos realizan su vida a diferentes escalas según la experiencia del sujeto involucrado. Evidentemente, el patrimonio agroecológico urbano de América Latina posee un legado histórico cultural a rescatar y conservar para el desarrollo humano de las generaciones actuales y posteriores.

\subsection{Reconocimiento al paisaje patrimonial agroecológico: recurso paisajístico de valores sociales, ambientales y culturales}

El patrimonio agroecológico urbano incorpora la agricultura urbana a un entorno y legado del que se creía ajena pues esta actividad se relacionaba más con los ámbitos naturales y rurales. Desde esta línea de pensamiento, se citan experiencias latinoamericanas donde expresan el legado de la agricultura a la ciudad y el reconocimiento del paisaje agroecológico en la metrópoli como recurso paisajístico.

$$
\text { Dichas experiencias }
$$

muestran

componentes para un reconocimiento susceptible de patrimonialización agroecológica urbana latinoamericana en México, Brasil y Colombia. Entonces, se percibe el valor de estos elementos relacionados con el desarrollo sustentable y la gestión ambiental; se han referenciado por su uso paisajístico como elementos construidos (infraestructura y herramientas) y elementos generadores (resilientes).

La infraestructura erigida en la agricultura urbana precisa la finalidad de la creación del espacio productivo con lo que se asegura el cuidado y acceso de los elementos fundamentales para la producción como el agua, la tierra, las semillas, la composta etc., lo que representa el "valor construido del paisaje". Además la sinergia generada asegura la resiliencia y la multifuncionalidad del paisaje donde lo ideal sería que fuera desde una perspectiva de sustentabilidad que defina su construcción. Esto permite promover funciones ecológicas, educativas, productivas, recreativas, participativas, económicas, y de compostaje, por mencionar algunas.

El "valor resiliente" de los procesos productivos agroecológicos detona la ciclicidad de la siembra y restablecimiento del suelo que cuida el crecimiento de plantas y frutos. Así nutre los ámbitos socioambientales y económicos al restablecer los vínculos humanos con la tierra a través de la alimentación; se crean redes sociales que establecen un sistema cuya finalidad es la conservación y la generación de esos y otros elementos que detonan más sistemas agroalimentarios en base a las condiciones ambientales y sociopolíticas que lo retroalimentan.

Con estos dos valores se establece la condición para patrimonializar al paisaje agroecológico urbano. Coincide con la propuesta de Armenta (2009, pp. 8-9) pues establece que "la recuperación, conservación y aprovechamiento del patrimonio cultural urbano es un paradigma de las políticas públicas en América Latina, en el cual es posible advertir tendencias y problemáticas comunes"; establece cinco "elementos justificativos de patrimonialización" a partir de las evidencias documentales donde muestran los atributos de la agricultura urbana, que son susceptibles de apreciar en el paisaje patrimonial agroecológico urbano: “(1) nuevas formas de actuación y gestión integrales; (2) la participación ciudadana; (3) imaginarios urbanos que exploran las diferentes formas en que es percibido y utilizado un mismo objeto o lugar patrimonializado por diversos estratos sociales; (4) recuperación del patrimonio urbano; y (5) un patrimonio urbano vivo y habitado" (Armenta, 2009, p. 10).

Desde una multiperspectiva que lleva a descifrar las formas de explorar el territorio y dar cabida a la agricultura urbana en las dinámicas de la metrópoli latinoamericana se muestra el carácter multifuncional y multipaisajístico de lo agroecológico urbano latinoamericano, donde es esencial señalar el valor construido que da pie al uso del espacio y potencial resiliente como recurso paisajístico sinérgico y transformador. Como ejemplo tres paisajes agroecológicos urbanos: Huerto Lomas del Sur "Mercado orgánico" en 
Paloma Gallegos Tejeda

Pedro Lina Manjarrez
Por un modelo de patrimonialización

del paisaje agroecológico urbano en América Latina
Tlajomulco de Zúñiga (perteneciente al Área Metropolitana de Guadalajara - AMG), Jalisco, México, con énfasis en su valor construido; "Revolução dos Baldinhos" de Florianópolis, Brasil en su valor resiliente; y Jardín Botánico de Bogotá, Colombia en la transición de los dos valores.

Estas experiencias ofrecen una respuesta a la expansión urbana anteponiendo las ideas de las dimensiones de la sustentabilidad como elementos justificativos de patrimonialización; presentando soluciones a problemáticas del deterioro de espacios urbanos, convirtiéndolos en un potencial ambiental productivo: atendiendo cuestiones de alimentación, salud, manejo de residuos y generación de capacidades autogestivas en la comunidad. Además, se observa una inercia institucional con la comunidad para dar seguimiento a los proyectos colectivos o en una apropiación de ellos.

\subsection{Huerto Lomas del Sur "Mercado orgánico" Tlajomulco de Zúñiga, Área Metropolitana de Guadalajara (AMG), Jalisco, México: infraestructura patrimonial agroecológica para la producción alimentaria}

El Huerto del Lomas del Sur "Mercado orgánico" se encuentra en una de las zonas donde el paisaje rural y agrícola del municipio de Tlajomulco de Zúñiga, sur del AMG, se transformó con el establecimiento de 51 nuevos fraccionamientos entre los años 2003 y 2014. La zona "Concepción del Valle - Cerro del Gato" se caracteriza por estar densamente poblada, ya que tiene 410,077 habitantes y un total de 133,976 hogares (Gobierno de Tlajomulco, 2018). Tan solo en el fraccionamiento Lomas del Sur se encuentran 21,026 habitantes y 6,739 casas. Con lo anterior se observa un municipio que aumentó 6 veces su población en los últimos 10 años.

Con el surgimiento de este cambio del paisaje rural a paisaje urbano, se establece un nuevo patrón de ocupación de uso del suelo periurbano en Tlajomulco de Zúñiga. Ahora bien, la adquisición de terrenos rurales mantiene un potencial en la tendencia de continuidad de conurbación espacial y territorial del AMG, con los consiguientes problemas sociales, ambientales y económicos de la población local.

El proyecto del Huerto Lomas del Sur se establece a partir de la recuperación de un espacio público que por abandono generaba inseguridad en la colonia. Este lugar es administrado por el municipio actualmente; trabaja en su mayoría el área productiva y cuentan con 10 voluntarios de la comunidad quienes han adoptado cada uno, una cama productiva. Reciben visitas escolares, y a partir de la promoción del "huerto comunitario" establecen huertos escolares, si las escuelas lo solicitan. Al mismo tiempo imparten talleres y pláticas sobre agricultura urbana al público en general. Las actividades de comercialización no se han llevado a cabo.

El Huerto Lomas del Sur "Mercado orgánico", tiene un área de 7,600 m2: su valor a primera vista es en la infraestructura que consta de una estructura circular metálica de exposición, salón didáctico, barra comunitaria, bodega, módulos de baños, barda perimetral, salón de usos múltiples de $100 \mathrm{~m} 2$, oficina, domo geodésico y cercos de camas de cultivo de $892.88 \mathrm{~m} 2$ en forma de "Mandala". El mercado también cuenta con almacén de herramienta, cuarto de composteo y áreas de cultivo (Gobierno de Tlajomulco, 2017).

Este paisaje agrourbano, para el gobierno municipal del periodo 2015-2017 fue emblemático, ya que buscaban mostrar que con voluntad política se recuperan los espacios públicos y se genera infraestructura para sistemas agroalimentarios donde se privilegia el autoconsumo, la producción y el comercio. El espacio resulta atractivo para visitar por su diseño, con lo que se potencializan procesos de enseñanza-aprendizaje sobre distintas formas de producir alimentos en la ciudad con la aplicación de ecotecnias.

El diseño es otra opción paisajística, tanto por lo que fue el lugar - espacio público abandonado donde se sufrían robos - (fig. 1), como por los materiales que se elaboraron para cubrir sus necesidades específicas como: puestos móviles de venta de productos (fig. 2), jaulas para las gallinas ponedoras, camas de producción biointensiva (fig. 3), el sistema de lombricompostaje y las lámparas de recarga de electricidad con fuerza eólica.

$\mathrm{Al}$ inicio el proceso de formación $\mathrm{y}$ capacitación de agricultura urbana buscaba establecer varias etapas de apropiación del lugar con la comunidad a través del cultivo de alimentos y el aprovechamiento de todos los espacios disponibles; este proceso participativo en el cambio de administración quedó limitado.

El proyecto arrancó con las ferias agroecológicas. En la primera, llevada a cabo cuando todavía era un área sin construir, el presidente municipal Alberto Uribe Camacho enfatizó que se implementarían políticas públicas en favor de la producción orgánica, "lo que sigue después del mercado, es crear una marca de productos orgánicos 
Paloma Gallegos Tejeda

Pedro Lina Manjarrez
Por un modelo de patrimonialización

del paisaje agroecológico urbano en América Latina
'Tlajomulco Orgánico"' (Gobierno de Tlajomulco, 2017 , p. 1). Para terminar, recalcó que "el objetivo de esta Feria Agroecológica es fortalecer las dinámicas de agroecología urbana, periurbana y campesina del Municipio de Tlajomulco de Zúñiga en el AMG, fomentando la participación de dinámicas de producción orgánica” (Gobierno de Tlajomulco, 2017, p. 1).

Quizás tenían nociones del impacto positivo que se estaba generando, pero no se lograron las condiciones necesarias para garantizar lo que se proponían. Las ferias no continuaron, lo que muestra que la propuesta de vincular al paisaje agroecológico urbano, con otras zonas productivas rurales tiene que garantizarse a pesar de los cambios administrativos. La comercialización entonces se convierte en un recurso paisajístico resiliente articulador de actores que ayuda a la valoración, en este caso, de los productos agrícolas locales.

Figura 1 - Paisaje urbano: espacio público en rescate; patio central del Huerto de Lomas del Sur antes de sembrar (2017)

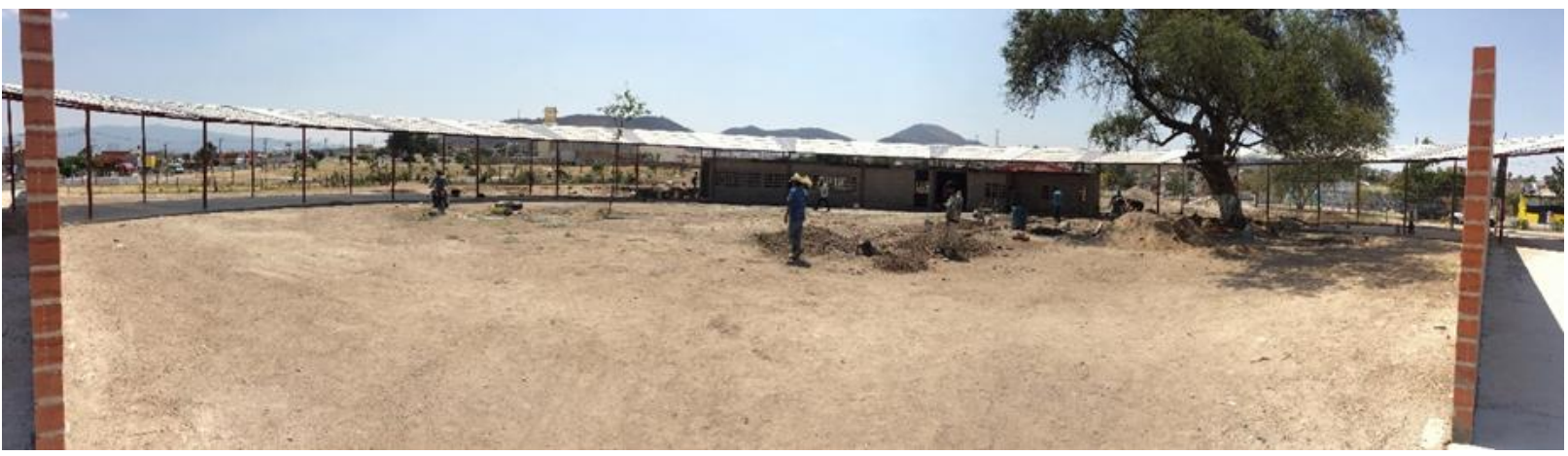

Fuente: fotografía Gallegos (2019). Huerto Lomas del Sur “Mercado Orgánico” Tlajomulco de Zúñiga, Jalisco. Paisaje agroecológico urbano. Acervo Investigación IPN.

Figura 2 - Puesto móvil de venta de productos

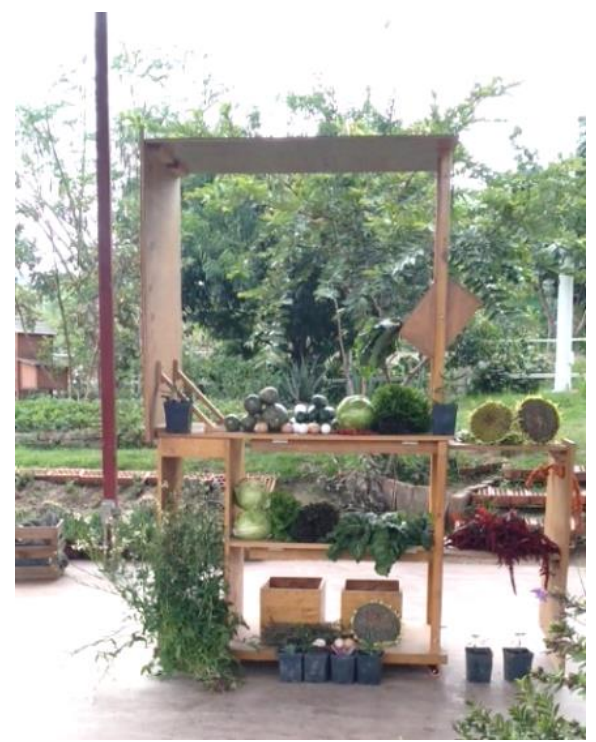

Fuente: fotografía Padilla (2019). Huerto Lomas del Sur "Mercado Orgánico" Tlajomulco de Zúñiga, Jalisco, México. Paisaje agroecológico urbano. Acervo Investigación IPN.
Figura 3 - Diseño circular, camas de producción biointensiva y salón de usos múltiples

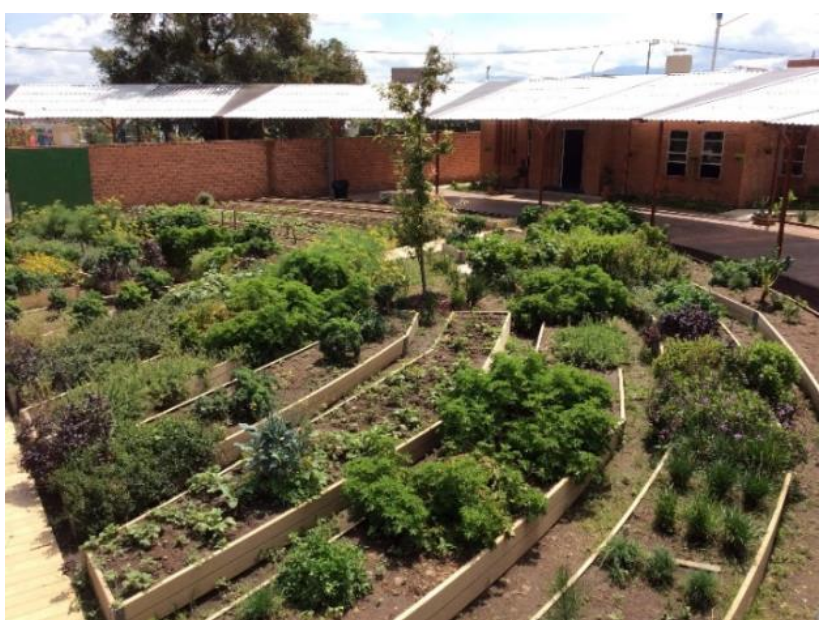

Fuente: fotografía Gallegos (2019). Huerto Lomas del Sur "Mercado Orgánico" Tlajomulco de Zúñiga, Jalisco. Paisaje agroecológico urbano. Acervo Investigación IPN. 
Además, es una experiencia donde las personas o grupos organizados pueden adoptar una parcela, cuidar el huerto y aprender técnicas de producción de alimentos para replicar lo aprendido en sus casas (Gobierno de Tlajomulco, 2018).

La inauguración del mercado orgánico fue el 21 de febrero de 2018, donde informaron que se invirtió en su construcción \$4.2 millones de pesos mexicanos. En el discurso inaugural del presidente municipal se reflejaron contexto, alcance y definición del proyecto ya que cuando se estaba elaborando sólo se partía de supuestos e impactos que este generaría en la zona.

Al mercado orgánico en el discurso inaugural lo definieron como:

(...) un espacio comercial donde se promueve un sistema de desarrollo comunitario, basado en la interacción positiva entre los vecinos y el medio ambiente dentro de un marco de sustentabilidad. En él se comercializan productos locales y artesanales donde se fomenta una cultura del cuidado del medio ambiente, una alimentación sana y un consumo responsable (Gobierno de Tlajomulco, 2018. p. 1).

Se explica el multipropósito como parte del valor construido del paisaje patrimonial agroecológico del huerto Lomas del Sur "Mercado orgánico" como usos diferentes: la tienda con 20 puestos móviles utilizados por productores y artesanos; la escuela abierta para todos los interesados con capacitaciones orientadas hacia la agroecología y educación ambiental y el huerto para generar un espacio productivo de corresponsabilidad donde se trabaja en conjunto con la comunidad.

\section{4. "Revolução dos Baldinhos" de Florianópolis, Brasil: patrimonio agroecológico resiliente para la transformación social}

El proyecto de gestión comunitaria de residuos orgánicos y agricultura urbana es parte del paisaje agroecológico urbano en la colonia Chico Mendes de la ciudad de Florianópolis, en el Estado de Santa Catrina, Brasil, y se llama "Revolução dos Baldinhos". La propuesta se origina en la zona continental de la ciudad, donde viven varias familias en situación de pobreza, baja escolaridad, inseguridad y desnutrición.

En 2007, tuvieron un problema por leptospirosis por contacto con ratas atraídas por la gran cantidad de basura acumulada en las calles. La solución a esta problemática fue el compostaje comunitario, base del proyecto. Este fue iniciativa del Centro de Estudios y Promoción de la

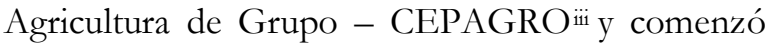
con una reunión en la colonia Chico Mendes.

La propuesta, consistió en que a través del compostaje termofílico (proceso donde a través del calor los residuos orgánicos se transforman en fertilizante natural) se controlaría el número de ratas. La transferencia de dicha técnica, donde los involucrados la aprendieron y dominaron llevó tiempo. Al arranque la participación fue limitada; esto cambió cuando la gente comprendió de qué se trataba el proyecto.

La experiencia mostró que el proceso de participación y autogestión alcanzadas por la comunidad bien podrían contribuir a la patrimonialización agroecológica urbana con el valor resiliente de las capacidades desarrolladas por la comunidad en el manejo de residuos y el grupo coordinador con la elaboración de la composta. Al observar el "Mandala" (fig. 4) se alcanzó el objetivo de vislumbrar el paisaje que se quería dibujar. La colonia unida por unos baldes; parada y sostenida por un huerto urbano fertilizado por la composta elaborada a partir de sus residuos orgánicos y que, al convertirse en abono, transformó la comunidad de algo aparentemente disfuncional por su situación de pobreza y violencia, en una comunidad vibrante y activa.

Figura 4 - Valor resiliente: Paisaje simbólico, Mandala del proyecto de agricultura urbana, "Revolução dos Baldinhos"

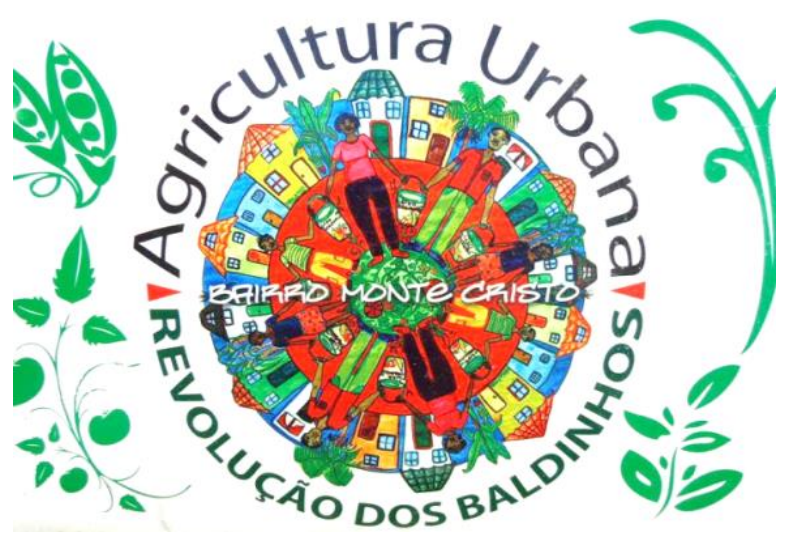

Fuente: fotografía Gallegos (2014). CEPAGRO, Universidade Federal da Santa Catarina, Florianópolis, Brasil.

Paisaje agroecológico urbano. Acervo Investigación CIIEMAD - IPN. 
Paloma Gallegos Tejeda

Pedro Lina Manjarrez
Por un modelo de patrimonialización

del paisaje agroecológico urbano en América Latina
Esta proyección habla del recurso resiliente que conforma un paisaje en consolidación cuyo elemento indispensable es la comunidad. Sin embargo, el tema de la participación ciudadana llevó tiempo. Este proyecto se consolidó y creció gracias al apoyo de cinco agentes comunitarios de compostaje de la misma localidad, quienes capacitaron a los vecinos en el manejo y separación correcta de residuos.

La logística resultó sencilla: distribuyeron baldes en las casas de los vecinos para recolectar los residuos orgánicos; los agentes comunitarios recogieron los baldes dos veces por semana para llevarlos al centro de compostaje. Una vez transformados, regresaron como fertilizante orgánico (composta) a la comunidad para usarlo en los huertos. Lograr esto fue reflejo del trabajo continuo de talleres y conferencias sobre alimentación e higiene con la habilitación de huertos familiares y comunitarios en distintos espacios de la colonia (Abreu, 2013).

Lo que se perfilaba como contingencia sanitaria, se transformó en un paisaje agroecológico urbano de autogestión comunitaria y de mejora de las condiciones de vida de las personas que se involucraban. Así se entiende la resiliencia con la integración de elementos que contribuyen a concebir el paisaje patrimonial agroecológico urbano: a través de la participación de los actores sociales con impacto en el ámbito socioambiental y la autogestión comunitaria (fig. 5).

La "Revolução dos Baldinhos" evolucionó a tecnología social, que se define como "los productos, técnicas o metodologías replicables, desarrolladas por la interacción con la comunidad $y$ que representan efectivas soluciones para la transformación social" (Peyloubet et al., 2009, p. 7). Esta tecnología social fue dada de alta en el Banco de Tecnología Social (BTS) de la base de datos de la Fundación del Banco de Brasil y ganó el premio de Tecnología Social por la Fundación en el 2013.

Para ese año la "Revolução dos Baldinhos" ya había beneficiado a 200 familias con la colecta de los residuos orgánicos, reciclado 500 toneladas de residuos orgánicos y registraron 1,550 participantes en el proyecto entre escuelas, niños, ONGs y asociaciones civiles. La mejora de la autoestima, la salud y la nutrición de la población son los resultados obtenidos (Abreu, 2013).
Figura 5 - Valor resiliente: Elaboración de composta en camas de compostaje termofílico

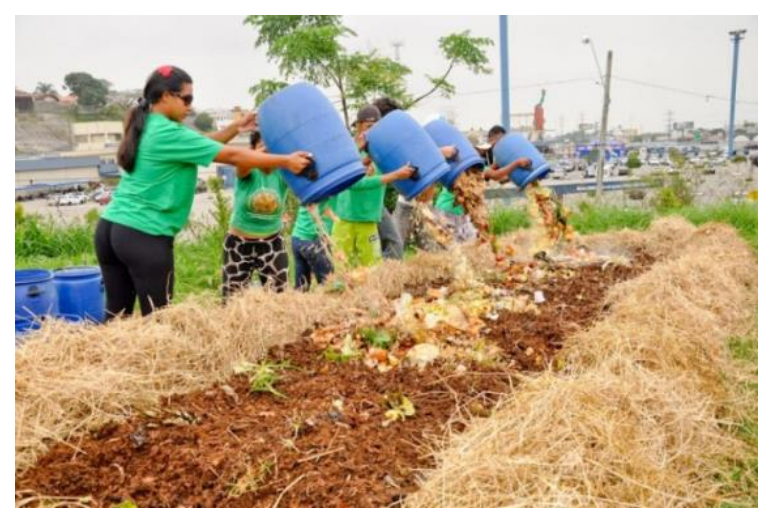

Fuente: CEPAGRO (2018a).

Una evidencia que muestra el valor resiliente de esta experiencia es que, en 2015, 2016 y 2017, esta acción agroecológica aplicada a un problema de salud formó parte de la Formación en Gestión de Residuos Sólidos de la Comunidad con participantes de todo el país. En 2016 se inició la replicación de este proyecto en un desarrollo inmobiliario de São Paulo y se ha condensado la experiencia en manuales y videos para su implementación (CEPAGRO, 2018a).

Cabe mencionar que para participar como agente comunitario los jóvenes a partir de los 16 años pueden involucrarse en el proyecto y recibir apoyo monetario. Esto, sólo si demuestran no haber consumido algún tipo de droga (por la condición de marginalidad problemática que persiste en la zona), lo que originó otra dinámica propia de ese grupo social, los "versos al viento" de la cultura del hip hop.

Maicon William de Jesus Chávez, agente comunitario de la "Revolução dos Baldinhos" escribió un Rap que resalta elementos patrimoniales intangibles y resilientes de este paisaje agrourbano, cuando en la letra dice:

\section{(...) hemos salido en la TV, representado a Brasil en Italia; en Rio de Janeiro y en Brasilia recibimos un premio; estamos juntos y seguimos de pie; la conciencia cambia, los niños son la esperanza y la Revolución ayuda a un mundo mejor. Lleno de árboles y flores se construye un futuro diferente; Mira lo bello que es la fuerza, por lo que podemos luchar, todos unidos esto se puede rescatariv (Chávez, 2016).}

Esta experiencia muestra los valores resilientes del patrimonio intangible del paisaje agroecológico urbano reflejado en testimonios, expresiones artísticas y dinámicas sociales que ha generado. Los testimonios son de personas que no sabían soñar hasta que la composta les invitó a hacerlo. 
Paloma Gallegos Tejeda

Pedro Lina Manjarrez
Por un modelo de patrimonialización

del paisaje agroecológico urbano en América Latina
2.5. Potenciación del valor de uso y resiliencia del patrimonio del Paisaje agrourbano en el Jardín Botánico de Bogotá, Colombia

Durante la administración 2004-2007 del Gobierno de Bogotá, estableció el programa de agricultura urbana "Bogotá Sin Hambre", de forma articulada con el Plan de Desarrollo de la ciudad, incidiendo en tres ejes el social, el regional, y de reconciliación. En este documento el gobierno reconoció la importancia económica de la agricultura urbana por su factibilidad; como actividad laboral para las familias desplazadas a causa de la violencia que se contabilizaban en cientos de miles para ese momento.

En el contexto urbano, los índices de pobreza, desnutrición y malnutrición de la población urbana se incrementaron y lo relacionaron con el crecimiento desorganizado de la ciudad y los distintos grupos sociales que se conformaron a causa de la migración, lo que se reflejó en diversas configuraciones culturales. En este contexto se determinó que la agricultura urbana podía ser un mecanismo de subsistencia para los nuevos habitantes (Gobierno de Bogotá, 2004, 2019).

De este proyecto de agricultura urbana, resultó la articulación con el Jardín Botánico "José Celestino Mutis". Desde un principio se tomaron en cuenta las diversas formas de "ocupación y producción” de la agricultura urbana que dependían de las condiciones locales del espacio donde se implementan.

Si a lo anterior se sumaba el potencial del valor construido del paisaje patrimonial que tiene un espacio como un jardín botánico, la oportunidad para el desarrollo del proyecto tendría elementos particulares que podrían reforzar el programa de agricultura urbana y su patrimonio botánico, resiliente y social. Así, la administración distrital logró integrar y presentar el proyecto de agricultura urbana desde el jardín botánico como una alternativa socioambiental con tres esferas de acción "Hábitat y Ambiente; Seguridad Alimentaria y Nutricional; y Desarrollo Humano Sostenible".

El Jardín Botánico "José Celestino Mutis" se vinculó al programa de "Bogotá Sin Hambre" pues reconoció que la agricultura urbana es "una estrategia que aporta conocimiento de la flora y agrobiodiversidad del distrito capital, capacitando a la población para el manejo de semillas y mejora de la cobertura vegetal para su conservación" cuyo objetivo fue promover y liderar las acciones de seguridad alimentaria y nutricional de la población con énfasis en la más pobre y vulnerable (Gobierno de Bogotá, 2019).

En esta experiencia un recurso patrimonial del paisaje agrourbano que se observa es el de la sinergia que genera resiliencia, ya que se cuenta con un bien como el jardín botánico que genera un valor extra al construido con otro medio paisajístico patrimonial vivo y toma mayor fuerza el valor del modo que en este caso es agroalimentario.

Por lo tanto, Lara (2008) cita a Paredes quien explica que el proyecto continúa ligado a otra serie de componentes como el Plan Maestro de Abastecimiento de Alimentos - PMAA, con el que también se abordan acciones estratégicas como la inclusión y la competitividad económica para la ciudad.

Entre los objetivos del programa de agricultura urbana "Bogotá Sin Hambre", que se cumplieron al vincularse con el jardín botánico se observaron los de:

Divulgar, promover y socializar los resultados generados en la investigación científica básica y aplicada sobre los usos potenciales, manejos agronómicos y sistemas de producción limpia de cada una de las especias seleccionadas y; Promover el uso de tecnologías limpias en los núcleos de formación, para que sean replicados por los beneficiarios del proyecto en diferentes escenarios de la ciudad (Lara, 2008, p. 45).

Sin redundar en la importancia que tienen los jardines botánicos en la difusión y concientización del valor resiliente del patrimonio botánico local, es pertinente para fines de este artículo, hablar de la gestión de este legado ya que se establece un vínculo con la sociedad que nos ayuda a observar el valor construido que este tiene. Por ejemplo, en el acervo botánico se encuentran camas productivas de quelites lo que conforma la diversidad en la belleza y abundancia de las hortalizas del paisaje agroecológico del Jardín Botánico de Bogotá (fig. 6) invita a explorar el paisaje agrourbano y mostrar la factibilidad de replicarlo en condiciones similares. 
Figura 6 - Valor resiliente y construido: Diversidad en la belleza de las hortalizas de quelites en el Paisaje agroecológico del Jardín Botánico de Bogotá
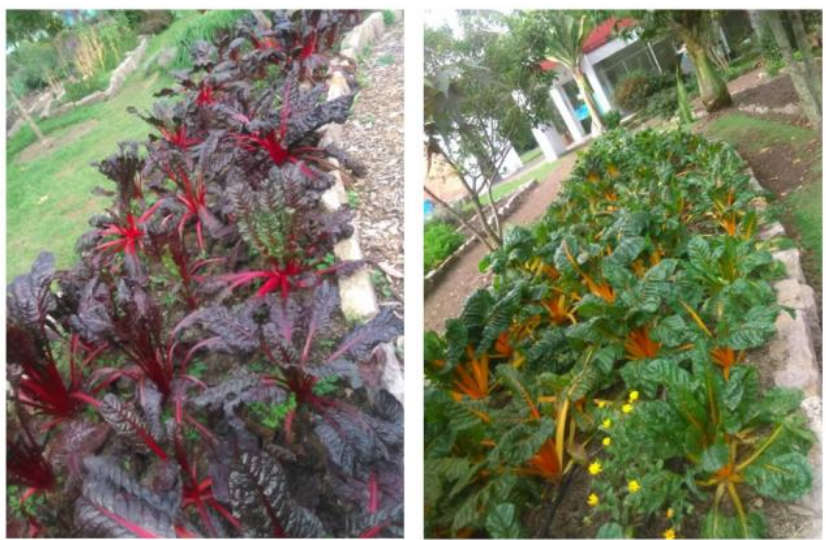

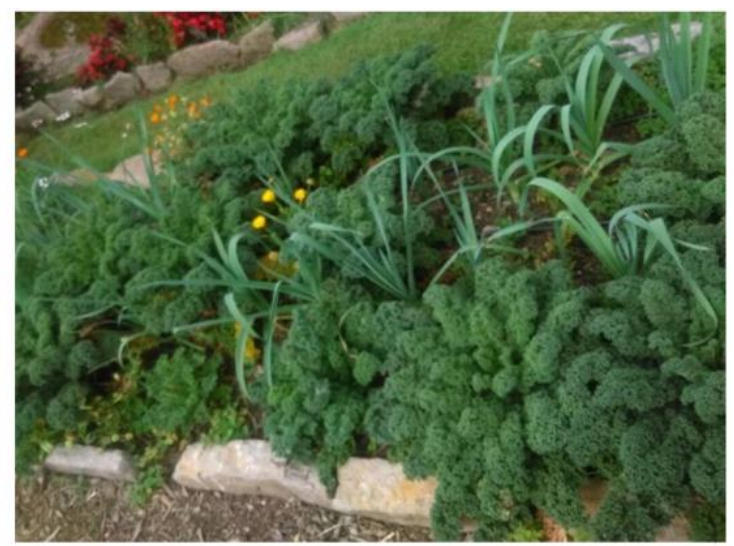

Fuente: fotografías Gallegos (2017). Jardín Botánico de Bogotá, Colombia. Paisaje agroecológico urbano. Acervo Investigación CIIEMAD - IPN.

Esta experiencia lleva a tomar conciencia del patrimonio que se está generando y la función social que tiene. $\mathrm{Al}$ mismo tiempo intenta reconocer que es un recurso que sufre constante cambio, esto le da una connotación de fragilidad por la dependencia que tiene a los insumos, los espacios y personas que lo producen.

Cuando se habla de fragilidad, Guglielmino (2007, p. 4) establece que el patrimonio se puede perder; por lo tanto, se debe promover un sentido de pertenencia social al patrimonio para perdurar y legarse a las generaciones futuras. Si bien, en su materialización original, pudieran ser diferente al actual esas transformaciones, que también son dignas de conservar.

En síntesis, las experiencias de vida en las tres ciudades latinoamericanas ponen en consideración el potencial patrimonial construido y resiliente desde su forma particular agroecológica para un paisaje agroecológico urbano latinoamericano. Los aportes de estas prácticas favorecen la producción alimentaria agroecológica urbana y al medio ambiente con el manejo de residuos y el rescate de espacios públicos; con los proyectos de agricultura urbana que promueven la creación de una comunidad consciente por una tierra cultivada transformadora del territorio. La metamorfosis de estos espacios con la creación de huertos, el acceso a alimentos frescos y de buena calidad, fomentan la educación agroalimentaria y ambiental, reflejo de la cooperación de los habitantes participantes.

El paisaje de huerto y jardines comestibles urbanos con participación social de los actores locales inducen a la percepción de un patrimonio agroecológico urbano, con la susceptibilidad de patrimonializar los bienes tangibles e intangibles del agro urbano. Resaltan los valores construidos en los lugares donde se desarrollan las experiencias, por otro lado, los valores resilientes vinculan a la comunidad y abren el camino para establecer una relación paisaje agrícola-urbano-medio ambientealimentación-desarrollo humano. Esta relación se observa en las transformaciones sociales $y$ ambientales que pretenden reflejarse de forma individual y colectiva en la mejora del acceso a los alimentos en la ciudad y la dieta familiar.

La diversidad de formas, saberes, herramientas, espacios, intereses, personas y objetivos que convergen en los espacios productivos alimentarios urbanos, crean una forma de potencial del paisaje patrimonial agroecológico urbano de amplio espectro. La clave es entender los avances y retrocesos que se tienen para lograr su consolidación y permanencia, ya que no depende solo de ciertos insumos, sino de una serie de voluntades y recursos de constante renovación.

\subsection{Patrimonialización del paisaje agroecológico urbano}

La patrimonialización es la acción de patrimonializar. Bustos (2004, p. 11) la establece como "un proceso voluntario de incorporación de valores socialmente construidos, contenidos en el espacio-tiempo de una sociedad particular, forma parte de los procesos de territorialización que están en la base de la relación entre territorio y cultura". Se trata de valorar individual y colectivamente referencias identitarias. Lo que crea una "conciencia 
Paloma Gallegos Tejeda

Pedro Lina Manjarrez
Por un modelo de patrimonialización

del paisaje agroecológico urbano en América Latina patrimonial", que representa los procesos sociales y culturales con los que se relaciona la sustentabilidad de su permanencia.

\subsection{Evidencias del potencial de los recursos paisajísticos agrícolas para un paisaje patrimonial agroecológico urbano}

En la idea del paisaje patrimonial agroecológico urbano se integran distintas dimensiones de la realidad además de incorporar acciones ambientales, sociales y económicas que den una perspectiva más integral del entorno en el que se vive: se trata de tener una visión de vida en la ciudad.

En su potencial de protección y conservación del legado agrícola que lo compone se identifican experiencias donde se establecen componentes del sentido de pertenencia de quienes establecen y cuidan al paisaje agroecológico urbano. Es un paisaje patrimonial construido que añade infraestructura al patrimonio urbano. Tiene la particularidad que dinamiza procesos productivos y organizativos de resiliencia, cuya finalidad es el establecimiento de sistemas agroalimentarios que vinculan desde el espacio público, escuelas, áreas verdes y hogares, en un sentido amplio hasta las zonas rurales y las Áreas Naturales Protegidas como fuente de recursos naturales, saberes campesinos y servicios ambientales para la ciudad.

El paisaje patrimonial agroecológico urbano añade conciencia y belleza a la ciudad; otorga elementos de arraigo e identidad a los agricultores urbanos que lo trabajan. El diseño, desarrollo y consolidación de este espacio natural se establece a partir de patrones innovadores de diseño con la intención de generar experiencias personales y colectivas de reflexión sobre el cuidado del entorno, de nosotros mismos y de los demás.

\subsection{Conservación del patrimonio paisajístico agroecológico urbano}

La conservación del paisaje agroecológico urbano es porque se quiere recuperar identidad y arraigo a través de los recursos paisajísticos alimentarios, lo que es una estrategia para la recuperación, transformación y embellecimiento del espacio público dando acceso a alimentos frescos y naturales. Por lo tanto, genera los elementos para vivir una experiencia con el espacio natural en lo urbano a través de la agricultura y la alimentación.
El paisaje agroecológico se conserva para establecer sistemas alimentarios metropolitanos que articulen al territorio a través del entorno natural y creen estrategias de autonomía y empoderamiento comunitario.

La conservación del paisaje agroecológico urbano se garantiza con la posesión del territorio, insumos, participación y el establecimiento de bancos de semillas y saberes en lugares estratégicos de la ciudad.

\subsection{Estrategia de recuperación: Conservación del sistema alimentario agrourbano}

El paisaje agroalimentario urbano es una expresión del recurso paisajístico agroecológico armónico que lo vincula con el campo y los recursos naturales. Lo cual concibe una perspectiva agrícola más amplia, territorialmente, dando lugar a la agrobiodiversidad, a acciones agroecológicas, al comercio justo, a la multifuncionalidad en los espacios productivos y alimentarios que impactan en el ambiente. Con miras en la articulación del territorio desde lo rural hasta las Áreas Naturales Protegidas que garantiza la retroalimentación del paisaje agroecológico urbano y que en conjunto resulta prioritaria para su conservación.

Además de la vinculación del paisaje agroecológico urbano con otros paisajes agrícolas y el reconocimiento de los elementos naturales de los que depende, son mecanismos para su conservación con la generación de infraestructura que abone a la mejora ambiental y escénica del lugar donde se establece. Armenta (2009, pp. 8-9) explica que el patrimonio urbano debe garantizar la permanencia del bien construido cuidando de no caer en la segregación socio espacial, evitando el despilfarro y la expansión urbana depredadora del ambiente, con la generación de un patrimonio urbano vivo, que ayude a la defensa de la ciudad.

García (2020, p. 12) establece que el juicio de amparo es el instrumento más usado para la conservación del patrimonio, ya que el acceso a la justicia en la defensa del territorio admite el interes legítimo por su preservación. Si bien "la metodología de la planeación modifica su proceder vertical y jerarquizado, y concilia las metas y los objetivos de las diversas entidades". Todavía prevalece la visión de desarrollo y manejo del territorio "desde afuera, planeando de arriba hacia abajo, o sea, desde las oficinas administrativas y no desde los lugares o con los lugares" (García, 2020, p. 4), lo que limita el arraigo. 
Paloma Gallegos Tejeda

Pedro Lina Manjarrez
Por un modelo de patrimonialización

del paisaje agroecológico urbano en América Latina
Para justificar ante la administración pública el establecimiento de esta infraestructura los mecanismos en la mayoría de los casos son confusos por carecer de lineamientos de creación. Sin embargo, el paisaje patrimonial agrourbano debe contar con espacio, infraestructura y recursos humanos que manejen y conserven el lugar.

Por ejemplo, en el Área Metropolitana de Guadalajara - AMG, del estado de Jalisco, la segunda metrópoli más grande de México, los mecanismos para justificar la construcción de infraestructura y la figura para asegurar el uso de suelo de agricultura urbana no existen, por consiguiente los elementos necesarios y el uso de suelo que se establecen deben relacionarse con otros rubros de desarrollo urbano.

Al hacer un ejercicio justificativo para desarrollar infraestructura de agricultura urbana en México, especificamente en el ÁMG, nos encontramos que:

(a) El Plan nacional de desarrollo habla de "autosuficiencia alimentaria y rescate del campo" en su apartado de proyectos regionales, sin referencia a la producción de alimentos en la ciudad;

(b) En los planes de desarrollo estatal tampoco se contempla la agricultura urbana; no obstante, por dar un ejemplo, en el plan estatal de desarrollo en Jalisco, se establece un programa de "Estados bajos en carbono", el cual opera el gobierno estatal en la Secretaria de Desarrollo Rural, y tiene como objetivo fomentar el aprovechamiento racional de los recursos naturales mediante el uso de tecnologías y sistemas de producción primaria amigables con el medio ambiente. Por entrar las ciudades en la cobertura geográfica de apoyo, la agricultura urbana es un rubro que puede recibir financiamiento;

(c) A nivel municipal, de los nueve planes de desarrollo municipales que conforman el AMG, se toma como ejemplo el plan municipal de desarrollo y gobernanza 2018-2021, del municipio de Tlajomulco de Zúñiga, municipio conurbado, donde se establece en el Índice 3. "Ciudad sustentable", apartado 3.6 la producción sustentable y consumo responsable, ahí se decreta como compromiso el derecho a la alimentación. El municipio se compromete a la promoción "de la agricultura urbana, periurbana y rural a pequeña escala con enfoque agroecológico" (Gobierno de Tlajomulco, 2019, pp. 39-40). Sin embargo, no se encontraron las formas de operar, tampoco el presupuesto ni las dependencias gubernamentales responsables de la ejecución de la actividad.
Bajo este panorama la conservación del paisaje agroecológico urbano depende más de una cuestión de voluntad política que como una estrategia para el desarrollo, lo que vulnerabiliza su permanencia.

Con la contextualización del paisaje agroecológico urbano, la clave para su habilitación, desarrollo y permanencia es su uso. Lo que muestra la importancia del involucramiento de las personas y el compromiso de quienes operan y gestionan los recursos para producir los alimentos. Así, conforme se consolida el paisaje agroecológico urbano se observan la diversidad de acciones, actores, formas, infraestructura y saberes que confluyen enriqueciendo los recursos paisajísticos del lugar.

Lo evidencia García (1999), con los usos sociales del patrimonio cultural, ya que este uso define la acción del estado para conservar el patrimonio con el fin de profundizar en la conciencia colectiva. Lo que se puede lograr a través del "patrimonio ambiental-natural urbano", por lo que hace énfasis que, para su conservación, hay que "crear condiciones materiales y simbólicas para que todas las clases sociales puedan compartirlo y encontrarle significado", para asegurar así su trascendencia (García, 1999, p. 19).

\subsection{Características para un modelo de patrimonialización del Paisaje agroecológico urbano}

La propuesta que emerge con la búsqueda de identificación y resguardo del paisaje patrimonial agrourbano es la reformulación de la política pública concerniente al desarrollo de la agricultura agroecológica urbana. A través del reconocimiento e inserción del paisaje agroecológico se busca la conservación de los sistemas alimentarios con un marco legal fundamentado en la participación de los actores sociales involucrados, en una producción limpia, de autoconsumo y comercialización, con miras a una mejora de la alimentación en su población local.

Por lo tanto, a partir de lo observado en el potencial de los recursos paisajísticos de los tres ejemplos latinoamericanos, se pensaría en las características de un posible Modelo de Patrimonialización del Paisaje Agroecológico Urbano que bien podría contener los siguientes aspectos generales:

(a) Reconocimiento del paisaje agroecológico urbano, a través de los componentes paisajísticos agrourbanos construidos y resilientes; 
Paloma Gallegos Tejeda

Pedro Lina Manjarrez
Por un modelo de patrimonialización

del paisaje agroecológico urbano en América Latina (b) Revisión del marco legal de patrimonialización local, regional y nacional;

(c) Inserción del paisaje patrimonial agroecológico urbano en planes de desarrollo, programas, reglamentos de todos los niveles gubernamentales;

(d) Generación de planes de manejo para la conservación del paisaje patrimonial agroecológico urbano.

Este planteamiento podría ser la toma conciencia del paisaje agrourbano vivo que se está creando, de cierta forma, la infraestructura debe acompañarse con un conjunto de actividades que lo mantengan rebosante, mostrando la unidad del patrimonio tangible con el patrimonio intangible.

\section{Consideraciones finales}

El paisaje patrimonial agroecológico urbano genera resiliencia en los lugares donde se establece, con lo que apoya al rescate y preservación del legado agrícola que lo compone. Esto se logra a través de reconocer sus elementos patrimoniales ecológicos y sociales que generan un sistema vivo de acceso a alimentos frescos en la ciudad. Es multifuncional y genera responsabilidad con la producción agroecológica hacía la integración del desarrollo humano y social en comunidades como de Bogotá, Tlajomulco de Zúñiga y Florianópolis. Los agricultores urbanos son quienes establecen sus componentes para asegurar su permanencia con un sentido de pertenencia y arraigo al cuidarlo.

El paisaje patrimonial agrourbano es un paisaje construido que añade infraestructura al patrimonio urbano. Tienen la particularidad de dinamizar procesos productivos y organizativos cuya finalidad es el establecimiento de sistemas agroalimentarios que vinculan el territorio. Es un testigo de la recuperación del espacio público a través de interacciones sociales detonadas por la alimentación.

El paisaje agroecológico urbano es un legado vivo a salvaguardar para la ciudad ya que conforma un territorio articulado donde las personas aumentan sus habilidades culturales, alimentarias, transformadoras hacia la sustentabilidad. Esto reviste una importancia de lucha social, ambiental, económica y cultural por la autosuficiencia alimentaria ante las desigualdades del incremento humano en las ciudades de América Latina.

El progreso y consolidación del paisaje patrimonial agrourbano, se establece a partir de pautas innovadores de diseño agroalimentario con la intención de generar espacios de experiencias personales y colectivas de reflexión-acción sobre el cuidado del entorno social y ambiental. Además de procesos de formación y capacitación que generen habilidades en la producción de alimentos, cuidado, nutrición del cuerpo con el acceso a alimentos. Añade conciencia ambiental, alimentaria y de belleza escénica a la ciudad, dando elementos de autonomía y autogestión a los agricultores urbanos que lo trabajan

La conservación del patrimonio del paisaje agroecológico urbano se acompaña de procesos de gestión que promueven en la ciudad el incremento de su espacio natural y el acceso a recursos alimentarios. Existe potencialmente un modelo de patrimonialización de acuerdo con las condiciones presentes en los casos latinoamericanos que muestran los caminos que buscan asegurar el patrimonio agroecológico urbano.

\section{Referencias bibliográficas}

Abreu, M. (2013). Gestão comunitária de resíduos orgânicos: o caso do Projeto Revolução dos Baldinhos (PRB), Capital Social e Agricultura Urbana - Florianópolis, Brasil. (Dissertação de Pós-graduação em Agroecossistemas, Centro de Ciências Agrarias). Universidade Federal de Santa Catarina, Florianópolis.

Altieri, M. \& Nihcolls, C. (2000). Agroecologia: Teoría y práctica para una agricultura sustentable. Serie Textos Básicos para la Formación Ambiental, 4 (pp. 5-124). Ciudad de México: PNUMA.

Altieri, M. (2010). El estado del arte de la agroecología: revisando avances y desafíos. In Vertientes del pensamiento agroecológico: fundamentos $y$ aplicaciones (pp. 77-104). Bogotá, Colombia: Universidad Nacional de Colombia.

Armenta, A. (2009). El patrimonio cultural urbano: Identidad, Memoria y Globalización. Andamios, 6(12), 7-10.

http://www.scielo.org.mx/scielo.php?script =sci_arttext\&pid=S187000632009000300001.

Bustos, R. (2004). Patrimonialización de valores territoriales: Turismo, sistemas productivos y desarrollo local. Aportes y transferencias, $8(2)$ 11-24. https://www.redalyc.org/pdf/276/2768020 2.pdf. 
Castillo, R. (2014). El Patrimonio Agrario: definición, caracterización y representatividad en el ámbito de la UNESCO. Boletín de la Asociación de Geógrafos Españoles (66), 105-124. https://www.agegeografia.es/ojs/index.php/bage/article/vie wFile/1782/1698.

Centro de Estudos e Promoção da Agricultura de Grupo. (2018a). Revolução dos Baldinhos chega a Taubaté (SP). CEPAGRO. https://cepagroagroecologia.wordpress.com /2018/03/10/revolucao-dos-baldinhoschega-a-taubate-sp/.

Centro de Estudos e Promoção da Agricultura de Grupo. (2018b). CEPAGRO e Revolução dos Baldinhos levam a gestão de residuos orgânicos para Foz do Iguacu (PR). CEPAGRO e Universidade Federal de Santa Catarina. https://cepagroagroecologia.wordpress.com /2018/07/11/cepagro-e-revolucao-dosbaldinhos-levam-a-gestao-de-residuosorganicos-para-foz-do-iguacu-pr/

Chávez, M. (2016). Novo Rap dos Baldinhos: Komay MC. Vídeo e Música Rap, Comunidade Chico Mendes). Florianópolis, Santa Catarina,

Brasil.

https://www.facebook.com/watch/?v=108 9503934445591 .

Erlij, M. (2004). Patrimonio, Ciudad y Sustentabilidad Urbana. Urbano, (11), 28-30. http:// revistas.ubiobio.cl/index.php/RU/art icle/view/522/485.

Erosion, Technology and Concentration Group. (2017). ¿Quién nos alimentara? La red campesina alimentaria o la cadena agroindustrial. $3^{\mathrm{a}} \mathrm{ed}$. Ciudad de México: ETC Group. http://www.etcgroup.org/sites/www.etcgro up.org/files/files/etc-quiennosalimentara2017-es.pdf.

García, N. (1999). Los usos sociales del Patrimonio Cultural. Cuaderno Patrimonio Etnológico: Nuevas perspectivas de estudio, 16-33. Instituto Andaluz del Patrimonio Histórico, Andalucía, España. https://www.iaph.es/export/sites/default/g alerias/documentacion_migracion/Cuadern o/1233838647815_ph10.nestor_garcia_cancl ini.capii.pdf.

García, E. (2020). Territorio y desafíos para la planeación en México. PatryTer, 3(6), 01-15. http://doi:10.26512/patryter.v3i6.26644.
Gobierno de Bogotá. (2019). Jardín Botánico de Bogotá. Programa de Agricultura Urbana. http://www.jbb.gov.co/.

Gobierno de Bogotá. (2004). Bogotá sin Indiferencia 2004-2008: Plan de Desarrollo Económico, Social y de Obras Públicas, Bogotá, D.C. Acuerdo 119 del 3 de junio de 2004. Planeación Distrital. https://www.shd.gov.co/shd/sites/default/ files/documentos/Plan $\% 20 \mathrm{de} \% 20$ Desarroll $\mathrm{O} \% 20$ -

$\% 20$ Bogot $\%$ C $3 \%$ A $1 \% 20 \sin \% 20$ Indiferencia -0.pdf

Gobierno de Tlajomulco. (2019). Plan municipal de desarrollo y Gobernanza del Municipio de Tlajomulco de Zúniga. Gobierno Municipal de Tlajomulco.

https://tlajomulco.gob.mx/sites/default/ file s/transparencia/reglamentos/AcuerdoPublic acion-

PlanMunicipalDesarrolloyGobernanzaTlajo mulco2018-2021-15Julio2019.pdf.

Gobierno de Tlajomulco. (2018). Tlajomulco inangura el primer mercado orgánico del municipio. Gestión Ambiental. Gobierno Municipal de Tlajomulco.

https://tlajomulco.gob.mx/noticias/tlajomu lco-inaugura-el-primer-mercado-organicomunicipal.

Gobierno de Tlajomulco. (2017). Se lleva a cabo la primera feria agroecológica Tlajomulco 2017. Gestión Ambiental. Gobierno Municipal de Tlajomulco.

https://transparencia.tlajomulco.gob.mx/pr ensa/se-lleva-cabo-la-primer-feriaagroecologica-tlajomulco-2017.

Guglielmino, M. (2007). La difusión del patrimonio: actualización y debate. Revista electrónica de Patrimonio Histórico, (1), 2-21. https://dialnet.unirioja.es/descarga/articulo /4013022.pdf

Hajba, G. (2017). Aspects to the Understanding of the Social Dynamics of Organic Food through the Example of a Community Garden in Budapest. Acta Ethnographica Hungarica, 62(2), 297-318. https://doi.org/10.1556/022.2017.62.2.3

Herrera, A. (2009). Impacto de la agricultura urbana en Cuba. Revista Novedades en Población, 5(9), 1-14.

http://www.novpob.uh.cu/index.php/Nov Pob/article/view/118/151.

Lara, A. (2008). Agricultura urbana en Bogotá: implicaciones en la construcción de una ciudad 
sustentable. (Monografía de Ciencia Política y Relaciones Internacionales). Pontificia Universidad Javeriana, Bogotá, Colombia. https://repository.javeriana.edu.co/bitstrea $\mathrm{m} /$ handle/10554/7809/tesis125.pdf?sequen $\mathrm{ce}=1$.

Mooney, P. \& ETC Group. (2019). La insostenible agricultura 4.0: digitalización y poder corporativo en la cadena alimentaria. Ciudad de México: Fundación Rosa-Luxemburg-Stiftung. https://www.etcgroup.org/sites/www.etcgr oup.org/files/files/la_insostenible_agricultu ra_4.0_web26oct.pdf

Mougeot, L. (2006). Cultivando mejores ciudades: agricultura urbana para el desarrollo sostenible. Centro Internacional de Investigaciones para el Desarrollo (Org.). Ottawa: Canadá. https://idl-bnc-

idrc.dspacedirect.org/bitstream/handle/106 25/34227/IDL-

34227.pdf?sequence $=12 \&$ is Allowed $=\mathrm{y}$

Organización de las Naciones Unidas para la Agricultura y la Alimentación. (2010). Agricultura "climáticamente inteligente": políticas, prácticas y financiación para la seguridad alimentaria, adaptación y mitigación. Roma, Italia: FAO. http://www.fao.org/3/i1881s/i1881s00.pdf.

Organización de las Naciones Unidas para la Agricultura y la Alimentación. (2006). La Agricultura Urbana y Periurbana en América Latina y el Caribe: Compendio de estudios de casos. Roma Italia: FAO. http://www.fao.org/ag/agp/greenercities/p df/Compendium.pdf.
Organización de las Naciones Unidas para la Agricultura y la Alimentación. (1996). El estado mundial de la agricultura y la alimentación. Roma Italia: http://www.fao.org/3/w1358s/w1358s07.h tm\#P3_41.

Peyloubet, P., Gargantini, D., O’Neill, T., Fenoglio, V., Valladares, G., Ortecho, M. \& Pasquale, F. (2009). Tecnología social y construcción colectiva del conocimiento. In XXVII Congreso de la Asociación Latinoamericana de Sociologia y VIII Jornadas de Sociologia de la Universidad de Buenos Aires. Asociación Latinoamericana de Sociología, Buenos Aires. http://cdsa.aacademica.org/000062/92.pdf

Rodríguez, A. (2020). Activación de un patrimonioterritorial mexicano: el amaranto en Santiago Tulyehualco, Ciudad de México. PatryTer, 3(6), 96-108. https://doi.org/10.26512/patryter.v3i6.2664 2

Silva, R. (2009). Agricultura, Paisaje y Patrimonio Territorial: los paisajes de la agricultura vistos como patrimonio. Boletín de la Asociación de Geografia Española, (49), 309$334 . \quad$ https://www.agegeografia.es/ojs/index.php/bage/article/vie $\mathrm{w} / 786 / 709$.

Silva, R. (2008). Hacia una valoración patrimonial de la agricultura. Scripta Nova, XII(275). http://www.ub.edu/geocrit/sn/sn-275.htm.

Toledo, V. (2014). Ecología y autosuficiencia alimentaria: hacia una opción basada en la diversidad biológica, ecológica y cultural de México. Ciudad de México: Siglo XXI.

\section{Notas}

i La Organización de las Naciones Unidas para la Alimentación y la Agricultura, ONUAA, o más conocida como FAO, por sus siglas en inglés "Food and Agriculture Organization", es un organismo especializado de la ONU que dirige las actividades internacionales encaminadas a erradicar el hambre.

ii El Grupo ETC - Grupo de Acción sobre Erosión, Tecnología y Concentración - monitorea el impacto de las tecnologías emergentes y las estrategias corporativas sobre la biodiversidad, la agricultura y los derechos humanos.

iii El CEPAGRO - Centro de Estudios y Promoción de la Agricultura grupal fue fundado en 1990, por pequeños agricultores y técnicos interesados en la formación de pequeñas redes productivas locales, como una forma de habilitar las propiedades rurales familiares de Florianópolis, Santa Catarina, Brasil.

iv Traducción de los autores. 